\title{
SPONTANEOUS DECAY OF THE EFFECTIVE COSMOLOGICAL CONSTANT
}

\author{
Murat Özer and M. O. Taha \\ Department of Physics, College of Science \\ King Saud University, P.O. Box 2455 \\ Riyadh 11451, Saudi Arabia
}

\begin{abstract}
We discuss the notion that quantum fields may induce an effective time - dependent cosmological constant which decays from a large initial value. It is shown that such cosmological models are viable in a non - de Sitter spacetime.
\end{abstract}

1. Introduction. The cosmological constant $\lambda$ in the Einstein field equations

$$
R_{\mu \nu}-\frac{1}{2} g_{\mu \nu} R=-8 \pi G T_{\mu \nu}+\lambda g_{\mu \nu}
$$

corresponds to the energy density for the vacuum. In the standard homogeneous and isotropic model of the universe described by the Robertson - Walker (RW) metric

$$
d s^{2}=-d t^{2}+a(t)^{2}\left[\frac{d r^{2}}{1-k r^{2}}+r^{2} \sin ^{2} \theta d \phi^{2}\right],
$$

where $k$ is the curvature constant and $a(t)$ is the scale factor of the universe, the field equations (1) give

$$
\left(\frac{\dot{a}}{a}\right)^{2}=\frac{8 \pi G}{3} \rho-\frac{k}{a^{2}}+\frac{\lambda}{3}
$$

where $\rho$ is the density of the universe. The controversy over the present value of the Hubble constant $\mathbb{H} H_{p}=\left(\frac{\dot{a}}{a}\right)_{p}$ continues with the two recently announced values of [1] $H_{p}=80 \pm 17 \mathrm{kms}^{-1} \mathrm{Mpc}^{-1}$ and [2] $H_{p}=52 \pm 8 \mathrm{kms}^{-1} \mathrm{Mpc}^{-1}$. The present value of the matter density $\rho_{M p}$ lies between $10^{-48} \mathrm{GeV}$ and $10^{-46} \mathrm{GeV}$ while the critical density $\rho_{p}^{c}=3 H_{p}^{2} / 8 \pi G \sim 10^{-47} \mathrm{GeV}$. Eq.(3) can be written as

$$
\frac{k}{H_{p}^{2} a_{p}^{2}}=\Omega_{p}+\Omega_{p}^{V}-1
$$

\footnotetext{
${ }^{1}$ We denote the present value of a quantity by the subscript $p$.
} 
where $\Omega_{p}$ and $\Omega_{p}^{V}$ are, respectively, the present values of the density parameter $\Omega=\rho / \rho^{c}$ and the vacuum density parameter $\Omega^{V}=\rho^{V} / \rho^{c}=\lambda / 3 H^{2}$ where $\rho^{V}=\lambda / 8 \pi G$. Since $H_{p}^{2} a_{p}^{2}$ is of order unity it follows that the right - hand side of (4) is either 0 (for $k=0$ ) or of order unity (for $k= \pm 1$ ). The present energy density estimates give $\Omega_{p} \sim 0.1-0.4$ from which we conclude that $\left|\Omega_{p}^{V}\right| \leq 1$. This implies $\left|\rho_{p}^{V}\right| \leq 10^{-47} \mathrm{GeV}$. However, gauge field theories imply that the value of the vacuum energy density $\rho^{V}$ was quite different in the early universe from its present value. For example, when the gauge symmetry $S U(2)_{L} \times U(1)_{Y}$ of the standard electroweak theory is broken by the vacuum expectation value of a Higgs doublet, the energy density stored in the broken vacuum is $\rho^{V}=-\frac{1}{8} M_{H}^{2}\langle\phi\rangle^{2} \sim-3 \times 10^{8} \mathrm{GeV}^{4}$ for a Higgs boson with mass $M_{H} \approx 200 \mathrm{GeV}$ and $\langle\phi\rangle=246 \mathrm{GeV}$. This is 55 orders of magnitude larger than the present bound. There may have occurred other spontaneous symmetry breakings in the early universe; the most celebrated of which is the grand unification symmetry breaking at a mass scale of $M \sim 10{ }^{16} \mathrm{GeV}$. The vacuum energy density associated with this breaking is $\sim 10^{64} \mathrm{GeV}$ which is 111 orders of magnitude larger than the present vacuum energy density. The cosmological constant problem $[3,4]$ is then to explain these huge orders of magnitude differences between $\rho_{\text {early }}^{V}$ and $\rho_{p}^{V}$ in a natural way; in particular without fine tuning the values of parameters to very many decimal places.

There have been various attempts at solving the problem of the cosmological constant some of which are reviewed in Ref.[4]. One line of attack that has not been reviewed in Ref.[4], is suggested in several studies [5 - 7] of quantum field theory in de Sitter space. These studies have found that the vacuum expectation value of the energy - momentum tensor $\left\langle T_{\mu \nu}\right\rangle$ of quantum fields in de Sitter space takes the form of an effective cosmological energy density times the metric tensor. Due to quantum instabilities the vacuum energy density (from now on we will denote $\rho^{V}$ by $\Lambda$ ) decays spontaneously and can dominate over the matter content of the universe. This idea has been examined in Ref.[8] which concludes that such quantum instabilities are unlikely to provide an effective cosmological energy density that decays from a large initial value. The analysis is based on the assumption that the spacetime metric is approximately de Sitter. This is a necessary condition for the decay mechanism of Refs.[5 - 7] to work. It is argued in Ref.[8] that this assumption cannot be avoided in a model that possesses a decaying effective vacuum energy density - or equivalently effective cosmological constant.

Shortly after the publication of Ref.[8] however, a decaying effective cosmological constant model was presented in Refs.[9]. Since then many extensions and generalizations of this model have been proposed [10 - 15]. Despite all the interest in this model and its extensions, an explanation of how any decaying - $\Lambda$ model circumvents the rather strong conclusions of Ref.[8] has not appeared in the literature. It is our purpose in this paper (1) to exhibit how the negative result of Ref.[8] has been avoided in the model of Ref.[9] and (2) to argue that decaying - $\Lambda$ models may arise in field theoretic contexts. 
Before introducing our analysis we would like to point out that, in the context of this paper, the term "effective cosmological constant" means the value of $T_{00}$ for a scalar field $\phi$ that represents the vacuum in a RW metric. This is the time - dependent vacuum energy density in this model. In a general quantum field theory in curved space - time one should calculate the effective potential and minimize it with respect to the matter fields to obtain the vacuum state. The value of the effective potential in this vacuum state is the effective cosmological constant. It should then be possible to directly relate this to the covariant vacuum energy momentum tensor, since both are defined in terms of the effective action in the vacuum state.

2. Formulation of the Problem. According to the findings of Refs.[5 - 7], the quantum instabilities in $\left\langle T_{\mu \nu}\right\rangle$ of the quantum fields which arise in a de Sitter space provide a variable cosmological constant which effectively solves the problem. To ensure a de Sitter - like universe which is a necessary condition for the gravitationally - mediated decay mechanism to work, Ref.[8] imposes the following two conditions on $\Lambda$ : (1) $\Lambda$ decays slowly and (2) $\Lambda$ always dominates over the matter and radiation content of the universe. It is then shown that, it seems improbable because of these two requirements that a realistic cosmology can be constructed which allows $\Lambda$ to decay from an initially large value.

To demonstrate the existence of decaying - $\Lambda$ cosmologies which evade the conditions mentioned above, we will examine them in the simplest variable $-\Lambda$ model presented in Refs.[9]. This model displays a decaying effective vacuum energy density (or equivalently a decaying effective cosmological constant) in a non - de Sitter context. The universe is neither born into a de Sitter phase nor does it evolve towards one. The model is based on the assumption that, in a general RW metric, the energy - momentum tensor $T_{\mu \nu}^{(U)}$ of the universe includes, besides $T_{\mu \nu}^{(m)}$ of matter, an additional piece $T_{\mu \nu}^{(V)}$ given by

$$
T_{\mu \nu}^{(V)}=-\Lambda(t) g_{\mu \nu} .
$$

The field equations then involve $\Lambda(t)$. One further specifies the model by requiring that the energy density of the universe is always given by its critical value $\rho^{c}=3 H^{2} / 8 \pi G$. This determines the intrinsic curvature $k$ to be unity and yields a definite $\Lambda(a)$ :

$$
\Lambda=\frac{3}{8 \pi G} \frac{1}{a^{2}} .
$$

In the radiation era, $p=\frac{1}{3} \rho$ and it is possible to solve explicitly for $a(t)$ :

$$
a(t)^{2}=a_{0}^{2}+t^{2},
$$

where $a_{0}$ is the scale factor of the universe at $t=0$. Thus the universe is born with an initial value $\Lambda_{0}=3 / 8 \pi G a_{0}^{2}$ for the vacuum energy density $\Lambda(t)$ which subsequently decays so that $\Lambda(t) \sim 3 / 8 \pi G t^{2}$ for $t \gg a_{0}$. The equation for $\dot{\Lambda}$ as a function of $\Lambda$ is

$$
\dot{\Lambda}=-2\left(\frac{8 \pi G}{3}\right)^{1 / 2} \Lambda^{3 / 2}\left(1-\Lambda / \Lambda_{0}\right)^{1 / 2} \text {. }
$$


For $t \gg a_{0}$ (i.e. $\left.a \gg a_{0}\right) \dot{\Lambda}$ may be well approximated by $\dot{\Lambda} \approx-\alpha \Lambda^{3 / 2}$ which is Eq.(2) of Ref.[8] with $\alpha=2 \sqrt{8 \pi G / 3}$.

It is clear from Eq.(7) that the spacetime metric is not de Sitter. The particular form of $\Lambda(a)$ in Eq.(6), which is charecteristic of this model and its generalizations, avoids these constraints. The resulting cosmology is realistic and phenomenologically viable [9]. The two conditions (1) $\Lambda \gg \rho$ for all $t$ and (2) $|\dot{\Lambda} a / \Lambda \dot{a}| \ll 1$, imposed in Ref.[8] as de Sitter conditions, are therefore not satisfied. In fact $\Lambda \gg \rho$ holds only when $a \approx a_{0}$ while $|\dot{\Lambda} a / \Lambda \dot{a}|=2$. One may thus conclude that the negative result of Ref.[8] is indeed limited to de Sitter - like metrics and does not extend to a general RW metric.

It thus remains to consider whether models of this type may arise in a field theoretic context. Consider a model in which the evolution of the universe is described by a uniform scalar field $\phi$. The metric is RW with $k=1$ and the Lagrangian density is specified as

$$
\mathcal{L}=-\frac{R}{16 \pi G}-\frac{1}{2} \partial_{\mu} \phi \partial^{\mu} \phi-V(\phi)
$$

where

$$
V(\phi)= \begin{cases}U(\phi) & , \quad 0 \leq \phi \leq \phi_{1} \\ V_{1} e^{-\gamma \phi} & , \quad \phi \geq \phi_{1}\end{cases}
$$

with $\gamma=-U^{\prime}\left(\phi_{1}\right) / U\left(\phi_{1}\right)>0$ and $V_{1}=U\left(\phi_{1}\right) e^{\gamma \phi_{1}}[16]$. The potential $U(\phi)$ may be chosen to have the characteristics required to spontaneously break the symmetry in $\phi \leq \phi_{1}$. We are interested in the large $t$ behavior of the model. For $\phi \geq \phi_{1}$, the field equations

$$
\left(\frac{\dot{a}}{a}\right)^{2}=\frac{8 \pi G}{3}\left(\frac{1}{2} \dot{\phi}^{2}+V(\phi)\right)-\frac{1}{a^{2}}
$$

and

$$
\ddot{\phi}+3 \frac{\dot{a}}{a}+V^{\prime}(\phi)=0
$$

admit the exact non - de Sitter solution

$$
a(t)=a_{2}(1+C t) \quad ; \quad \phi(t)=\phi_{2}+2 \gamma^{-1} \ln (1+C t)
$$

where

$$
C^{2}=\frac{3}{8 \pi G} \gamma^{2} a_{2}^{-2}\left(6-\frac{3}{8 \pi G} \gamma^{2}\right)^{-1} \quad ; \quad \phi_{2}=\gamma^{-1} \ln \left[\frac{2 \pi G}{3} V_{1} a_{2}^{2}\left(6-\frac{3}{8 \pi G} \gamma^{2}\right)\right],
$$

provided that $\gamma^{2} / 8 \pi G<2$. The constant rate $\dot{a}$ of the expansion is less than unity when $\gamma^{2} / 8 \pi G<1$. The solution (13) is valid for $t \geq t_{1}$ where $\phi\left(t_{1}\right)=\phi_{1}$. Eqs.(14) determine $C$ and $a\left(t_{1}\right)$ or equivalently $C$ and $a(0)$ if $U(\phi)$ is known. 
The scalar field $\phi$ may be taken either as the nonvanishing vacuum expectation value of a quantum field or as a representation of the non - trivial vacuum of curved spacetime. The induced effective vacuum energy density in either case is $\frac{1}{2} \dot{\phi}^{2}+V(\phi)$. This yields

$$
\Lambda(t)=6 C^{2} \gamma^{-2}(1+C t)^{-2} \sim 6 \gamma^{-2} t^{-2},
$$

for large $t$. Thus $\Lambda(t)$ decays to zero from any initially large value it might have had. One observes that $\dot{\Lambda}=-\sqrt{2 / 3} \gamma \Lambda^{3 / 2}$ as in Eq.(2) of Ref.[8] while $|\dot{\Lambda} a / \Lambda \dot{a}|=2$ as above. For large $t$ the scale factor $a(t)$ is not much different from that of the critical density model indicating that the two models have desirable cosmological features in common.

3. Stability Analysis. Before we discuss the stability of our solution, an important and novel feature of models with $\rho_{\phi}$ (or $\Lambda$ ) decaying as $a^{-2}$ should be pointed out. Using Eqs.(13)and (14), Eq.(11) can be cast into

$$
\left(\frac{\dot{a}}{a}\right)^{2}=-\frac{k_{e f f}}{a^{2}}
$$

where

$$
k_{e f f}=k-\frac{1}{\left(1-\frac{\gamma^{2}}{2 \chi^{2}}\right)}<0,
$$

with $k=1$ and $\chi^{2}=8 \pi G$. The parameter $k_{\text {eff }}$ is the effective curvature scalar of the present model universe. Thus, even though the three - geometry of the universe is closed (i.e. $k=1$ ), the universe evolves as if it is open due to the fact that $k_{\text {eff }}<0$. This always happens even with more realistic models containing radiation and matter whenever $\rho_{\phi}$ (or $\Lambda$ ) decays as $a^{-2}$. Consequently the universe avoids collapse as long as $k_{\text {eff }}$ remains negative. Hence the intriguing possibility of a closed but ever - expanding universe.

Stability of the system of equations (11) and (12) with $V(\phi)$ given in Eq.(10) can be investigated by introducing a new time variable $\tau$ and defining a new scale factor $\alpha(\tau)$ such that [17] (see also Ref.[18])

$$
\begin{aligned}
& \frac{d}{d t}=V^{1 / 2} \frac{d}{d \tau} \\
& a(\tau)=\exp (\alpha(\tau)) .
\end{aligned}
$$

In terms of these variables equations (11) and (12) become respectively

$$
\begin{gathered}
\alpha^{\prime 2}=\frac{\chi^{2}}{3}\left(\frac{1}{2} \phi^{\prime 2}+1\right)-\frac{k e^{-2 \alpha+\gamma \phi}}{V_{1}} \\
\phi^{\prime \prime}=\frac{1}{2} \gamma \phi^{2}-3 \alpha^{\prime} \phi^{\prime}+\gamma
\end{gathered}
$$


where a prime denotes differentiation with respect to $\tau$. An equation which does not depend on $k$ and $V_{1}$ is obtained by differentiating Eq.(19):

$$
\alpha^{\prime \prime}=\frac{1}{2} \gamma \alpha^{\prime} \phi^{\prime}-\frac{\chi^{2}}{3}\left(\phi^{\prime 2}-1\right)-\alpha^{\prime 2}
$$

Defining the new variables $x=\phi^{\prime}$ and $y=\alpha^{\prime}$ the system of equations (20) and (21) are transformed into the following two - dimensional autonomous system

$$
\begin{aligned}
& x^{\prime}=\frac{1}{2} \gamma x^{2}-3 x y+\gamma, \\
& y^{\prime}=\frac{1}{2} \gamma x y-\frac{\chi^{2}}{3}\left(x^{2}-1\right)-y^{2} .
\end{aligned}
$$

The system (22) possesses the following critical points

$$
\begin{aligned}
& (x, y)=\left( \pm 1, \pm \frac{\gamma}{2}\right) \\
& (x, y)=\left( \pm \frac{\gamma \sqrt{2}}{\sqrt{6 \chi^{2}-\gamma^{2}}}, \pm \frac{\chi^{2} \sqrt{2}}{\sqrt{6 \chi^{2}-\gamma^{2}}}\right) .
\end{aligned}
$$

In Figure 1, we depict the phase flow in the $x y$ plane for $\chi^{2}=3$, and $\gamma=1$, where the critical points corresponding to (23) and (24) are the attractor $A$ and the saddle point $S$, respectively. The $k=+1$ solutions are separated from the $k=-1$ solutions by the $k=0$ trajectories which are the hyperbolae $y^{2}=\frac{1}{2} x^{2}+1$, as follows from Eq.(19). (For a detailed discussion of the solutions for other values of the coupling we refer the reader to Ref.[18]) Figure 1 indicates that only some of the $k=+1$ solutions are asymptotically stable. Does our solution given in Eq.(13) belong to the family of the stable solutions? The answer is affirmative. As we have noted above cosmological solutions with $\rho_{\phi}$ decaying as $a(t)^{-2}$ lead to an effective curvature scalar given in Eq.(17). It is shown in Ref.[18] that irrespective of the value of the coupling all the $k=-1$ solutions are asymptotically stable. Hence the stability of our solution follows as long as $k_{\text {eff }}$ remains negative, which is the case for $\gamma^{2} / \chi^{2}<2$ ?

4. Primordial Nucleosynthesis. It is argued in Ref.[8] that cosmological models that are dominated by a decaying cosmological constant predict a primordial ${ }^{4} \mathrm{He}$ abundance today which is $<10^{-26}$ (Assuming that ${ }^{4} H e$ are not direct products of $\Lambda$ ). This conclusion follows from combinations of assumptions which do not hold in models of the type considered here. The details of primordial nucleosynthesis in decaying vacuum cosmologies with $\Lambda$ decaying as $a^{-2}$ have been considered in Ref.[14]. Here ,we will outline how the

\footnotetext{
${ }^{2}$ The stability of all the cosmological solutions with $\rho_{\phi}=\frac{1}{2} \dot{\phi}^{2}+V(\phi)$ decaying as $a(t)^{-2}$ and $V(\phi)=$ $V_{1} e^{-\gamma \phi}$ can be investigated by substituting in Eq.(11) $\rho_{\phi}=3 c / \chi^{2} a^{2}$, where $c>1$ so that $k_{e f f}$ in Eq.(17) is negative. One then finds that all the $k=+1$ solutions with this property are indeed stable. We hope to expand on this in future work.
} 
method of Ref.[14] can be implemented in a field - theoretic model. As the scalar field $\phi$ evolves and decays into radiation and matter, there comes an epoch at which the scale factor is no longer given by that in Eq.(13). This is due to the accumulation of radiation (i.e. relativistic particles) and matter (nonrelativistic particles). The period starting with this epoch, at which $t=t_{*}$ and $a=a_{*}$, may be called the radiation - dominated era, and lasts, as in the standard model, until the radiation and matter energy densities become equal at $a=a_{e q}$. The decay of the $\phi$ - field energy density into radiation and matter can be modeled by [19]

$$
\rho_{r}=\Gamma_{r} \dot{\phi}^{2}, \rho_{m}=\Gamma_{r} \dot{\phi}^{2}
$$

where $\Gamma_{r}$ and $\Gamma_{m}$ are very slowly varying functions of time. We approximate them by constants here. Upon the inclusion of radiation and matter Eq.(11) changes to

$$
\begin{aligned}
\left(\frac{\dot{a}}{a}\right)^{2} & =\frac{8 \pi G}{3}\left(\rho_{r}+\rho_{m}+\rho_{\phi}\right)-\frac{1}{a^{2}} \\
& =\frac{8 \pi G}{3}\left[\left(\Gamma_{r}+\Gamma_{m}+\frac{1}{2}\right) \dot{\phi}^{2}+V(\phi)\right]-\frac{1}{a^{2}}
\end{aligned}
$$

and Eq.(12), which follows from the conservation equation

$$
d\left[\left(\rho_{\phi}+\rho_{r}+\rho_{m}\right) a^{3}\right]+\left(p_{\phi}+p_{r}+p_{m}\right) d a^{3}=0
$$

when $\rho_{r}=\rho_{m}=0$, is replaced by

$$
\left(1+2 \Gamma_{r}+2 \Gamma_{m}\right) \ddot{\phi}+3\left(\frac{4}{3} \Gamma_{r}+\Gamma_{m}+1\right) \frac{\dot{a}}{a} \dot{\phi}+\frac{d V(\phi)}{d \phi}=0 .
$$

Due to the creation of radiation and matter the scalar field and its potential change to

$$
\phi(t)=\phi_{2}-\frac{2}{n \gamma} \ln (1+C t) \quad, \quad V(\phi)=D V_{1} e^{\gamma \phi}
$$

where the coefficients $n$ and $D$ are to be determined from Eqs.(26) and (28) self - consistently. We obtain the relations

$$
D=\frac{2}{n(n+1)} \quad, \quad \Gamma_{r}+\Gamma_{m}=n\left(\frac{n-1}{n+1}\right) .
$$

Since the kinetic energy of the scalar field cannot convert to radiation and matter with hundred percent efficiency, the sum $\Gamma_{r}+\Gamma_{m}$ must be less than unity. Hence, it follows from Eq.(30) that $n<1+\sqrt{2}$. Without loss of generality, we will take in the following $n=2$. Next, we express the scalar field energy density $\rho_{\phi}$ and the pressure $p_{\phi}$ in terms of $\Gamma_{r}+\Gamma_{m}$ and make the assumption that even though radiation and matter are in thermal equilibrium they evolve almost independently of each other, as in the standard model. 
Upon multiplying through by a the energy conservation equation (27) then leads to two approximately independent equations:

$$
\begin{aligned}
& d\left(\rho_{r} a^{4}\right)=\frac{21 \Gamma_{r}}{\chi^{2}\left(6-3 \gamma^{2} / \chi^{2}\right)} a d a, \\
& d\left(\rho_{m} a^{3}\right)=\frac{21 \Gamma_{m}}{\chi^{2}\left(6-3 \gamma^{2} / \chi^{2}\right)} d a .
\end{aligned}
$$

We assume that Eqs.(31) and (32) hold approximately during the entire evolution of the universe, probably with different values for $\Gamma_{r}$ and $\Gamma_{m}$ in different periods. Integrating these equations from $a$ to $a_{e q}$ results in

$$
\begin{aligned}
\rho_{r}^{R D} & =\frac{\omega_{e q}^{(r)} a_{e q}^{2} \chi^{-2}}{a^{4}}+\frac{\frac{21}{2} \Gamma_{r} \chi^{-2}\left(6-3 \gamma^{2} / \chi^{2}\right)^{-1}}{a^{2}}, \\
\rho_{m}^{R D} & =\frac{\omega_{e q}^{(m)} a_{e q}^{2} \chi^{-2}}{a^{3}}+\frac{21 \Gamma_{m} \chi^{-2}\left(6-3 \gamma^{2} / \chi^{2}\right)^{-1}}{a^{2}},
\end{aligned}
$$

where $\omega_{e q}^{(r)}=\chi^{2} \rho_{e q} a_{e q}^{2}-\frac{21}{2} \Gamma_{r} /\left(6-3 \gamma^{2} / \chi^{2}\right)$ and $\omega_{e q}^{(m)}=\chi^{2} \rho_{e q} a_{e q}^{2}-21 \Gamma_{m} /\left(6-3 \gamma^{2} / \chi^{2}\right)$. Due to the fact that majority of the existing particles are relativistic and that the scale factor is relatively small in the radiation - dominated era, the $a^{-4}$ term in Eqs.(33) dominate over the $a^{-} 2$ term. Thus, to a very good approximation near and at $T \approx 1 \mathrm{MeV}$ the radiation energy density reduces to

$$
\rho_{r}^{R D} \approx \frac{\omega_{e q}^{(r)} a_{e q}^{2} \chi^{-2}}{a^{4}}
$$

It should be noted that the radiation energy density in Eqs.(35) has the same dependence on the scale factor as in the standard model. Substituting Eq.(35) into Eq.(25) and neglecting $\rho_{m}$ and $\rho_{\phi}$, the scale factor is found to be

$$
a(t) \approx\left(\sqrt{\frac{4}{3} \omega_{e q}^{(r)}} a_{e q}\left(t-t_{*}\right)+a_{*}^{2}\right)^{1 / 2},
$$

where $t_{*}$ at which $a=a_{*}$ marks the epoch when the scalar field and the radiation energy densities are equal. The universe is assumed to enter the radiation - dominated era once $a>a_{*}$, and $a \gg a_{*}$ near and at $T \approx 1 \mathrm{MeV}$. Hence, the behavior of the scale factor is $a(t) \sim t^{1 / 2}$, just as in the standard model. The rest of the analysis of primordial nucleosynthesis is similar to that in the standard model. The details can be found in Ref.[14] where it is shown that the desired ${ }^{4} \mathrm{He}$ abundance is obtained in models with a vacuum energy decaying as $a^{-2}$.

5. Conclusions. The existence of decaying - $\Lambda$ models, in phenomenological or field theoretic contexts, indicates that the negative result of Ref.[8] hinges on the assumption that 
spacetime is de Sitter - like. Our conclusion is that it is not unlikely that a realistic quantum field cosmology with a decaying effective cosmological constant can be constructed. There is no general argument against such a construction. It is only necessary that the spacetime be non - de Sitter in such a theory. Cosmological models based on a homogeneous scalar field whose energy density behaves like a time - variable cosmological constant

in a non - de Sitter spacetime have long been considered. References [16] , [20 - 22], and [23] are examples of such an endeavor.

\section{References}

1. W. Freedman et al., Nature 371,757 (1994).

2. A. Sandage et al. ApJ, 423, 16 (1994).

3. L. Abbott, Sci. Am., 258, 106 (1988).

4. S. Weinberg, Rev. Mod. Phys., 61, 1 (1989).

5. N. Myhrvold, Phys. Rev. D28, 2439 (1983).

6. L. Ford, Phys. Rev., D31, 710 (1985).

7. E. Mottola, Phys. Rev., D31, 754 (1985).

8. W. A. Hiscock, Phys. Lett., 166B, 285 (1986).

9. M. Özer and M. O. Taha, Phys. Lett., 171B, 363 (1986) ; Nucl. Phys., B287, 776 (1987).

10. M. Gasperini, Phys. Lett., B194, 3406 (1987).

11. W. Chen and Y. S. Wu, Phys. Rev. D41, 695 (1990) ; Erratum Phys. Rev. D45, 4728 (1992).

12. D. Pavon Phys. Rev. D43, 375 (1991).

13. M. S. Berman, Phys. Rev. D43, 1075 (1991).

14. A. M. Abdel - Rahman, Phys. Rev. D45, 3497 (1992).

15. J. Matyjasek, Phys. Rev. D45, 4154 (1995).

16. Similar exponential potentials have been considered by several authors as inspired by string theory. See, e.g. M. Özer and M. O. Taha, Phys. Rev. D45, 997 (1992).

17. A. B. Burd and J. D. Barrow, Nucl. Phys. B308, 929 (1988).

18. J. J. Halliwel, Phys. Lett., B185, 341(1987).

19. Creation of matter due to the decay of the scalar field as modeled in Eq.(25) is similar to the mechanism in the power - law inflationary model of F. Lucchin and S. Matarrese, Phys. Rev. D32, 1316 (1985).

20. P. J. E. Peebles and B. Ratra, ApJ. 325, L17 (1988).

21. B. Ratra and P. J. E. Peebles, Phys. Rev. D37, 3406 (1988).

22. B. Ratra and A. Quillen, Mon. Not. R. Astron. Soc. 259, 738 (1992).

23. K. M. Mubarak and M. Özer, Clas. and Quant. Grav. 15, 75 (1998). 


\section{FIGURE CAPTIONS:}

Figure 1: Phase flow in the $x y$ plane for $\chi^{2}=8 \pi G=3$, and $\gamma=1$. The dashed curves are the hyperbolae $y^{2}=\frac{1}{2} x^{2}+1$ corresponding to the $k=0$ trajectories. The region between the two hyperbolae correspond to the $k=+1$ trajectories while the regions inside the hyperbolae correspond to the $k=-1$ trajectories. The points $A$ and $S$ are the attractor and the saddle points, respectively. 